\title{
Were Low Transferrin Levels Associated with ADHD Symptoms?
}

\author{
Rongwang Yang ${ }^{1}$, Suhan Zhang ${ }^{1}$, Rong $\mathrm{Li}^{1}$ and Zhengyan Zhao ${ }^{2}$ \\ 1Department of Child Psychology, The Children's Hospital, Zhejiang University School of Medicine, Hangzhou, Zhejiang Province, China \\ 2Department of Child Health Care, The Children's Hospital, Zhejiang University School of Medicine, Hangzhou, Zhejiang Province, China
}

\section{Were low transferrin levels associated with ADHD symptoms?}

In the December issue of the Journal, Kwon et al. ${ }^{1}$ addressed a valuable issue on the association between attention deficit-hyperactivity disorder (ADHD) and iron deficiency in Korean children. The results showed that there were significant differences found in transferrin, $\mathrm{MCV}$ and $\mathrm{MCH}$ between the children with ADHD and normal controls. In conclusion, the authors proposed low transferrin levels might be related to ADHD symptoms. ${ }^{1}$ However, we have several concerns that might affect the interpretation of the results. We would like to share them.

Firstly, iron storage is greatly associated with the nutritional status of the children and the socioeconomic status of the families. ${ }^{2}$ Therefore, the indicators of body weight, height and body mass index should be collected for both study group and controls. In addition, the detailed information of anual income of the families, educational levels of the parents and so on should also be recorded. Consequently, by doing that, it would be able to exclude the possible confounding factors and selection bias, and power the results with more strength. ${ }^{3}$

Secondly, a statistic difference was found in transferrin levels between the ADHD group and the normal controls, but did it have a clinical significance ${ }^{1}$ The study showed that transferrin levels were $248.42 \pm 44.15$ and $266.27 \pm 25.40 \mathrm{mg} / \mathrm{dL}$ for the ADHD subjects and controls, respectively. From Table 2 we can see that the reference value for transferrin is from 200 to $360 \mathrm{mg} / \mathrm{dL}$. The mean levels for both groups were in the interval, which suggested that they were in normal range. There-

\footnotetext{
Received: May 12, 2012 Revised: July 3, 2012

Accepted: July 3, 2012 Available online: November 14, 2012

$\triangle$ Correspondence: Rongwang Yang, MD

Department of Child Psychology, The Children's Hospital, Zhejiang University School of Medicine, No.57, Zhuganxiang Road, Hangzhou, Zhejiang Province, China

Tel: +8657188873304, Fax: +8657187033296

E-mail: colortea@zuaa.zju.edu.cn

(a) This is an Open Access article distributed under the terms of the Creative Commons Attribution Non-Commercial License (http://creativecommons.org/licenses/bync/3.0) which permits unrestricted non-commercial use, distribution, and reproduction in any medium, provided the original work is properly cited.
}

fore, it might be difficult to assert the statistic difference have clinical significance. In addition, the indicators of MCV and $\mathrm{MCH}$ were higher in ADHD group than the normal. Both of them had significant differences. But the authors did not discuss this result in details. We would like to receive any comments on it from the authors.

Finally, lack of teachers' ADHD symptoms rating scales was a limitation for the study. In the article by Kwon et al., Conners' Parent rating scale and Dupaul Parent ADHD rating scale only collected the information from parents (method section of the abstract), although there was the Computerized Attention Deficit-Hyperactivity Disorder Diagnostic System employed to test the executive function. As is known, ADHD is a disorder based on functional impairment not on symptoms alone. ${ }^{4-8}$ For children in primary school the most functionally impairing environment is the classroom. Therefore, only the symptoms evaluation from parents is not enough. Furthermore, without introduction of the blind methodology, the raters assessing the ADHD symptoms more subjectively resulted in bias of outcomes. So, we strongly suggest that teachers' AD$\mathrm{HD}$ rating scale should be used to assess the ADHD symptoms besides only the parents ADHD rating scale. Using comprehensive evaluation tools will judge the actual correlation between transferrin levels and ADHD symptoms, and reduce the bias by the expectancy from the researchers.

In considering the above mentioned concerns, we think that the authors should take more cautions in interpreting the results of this study.

\section{REFERENCES}

1. Kwon HJ, Lim MH, Ha M, Kim EJ, Yoo SJ, Kim JW, et al. Transferrin in Korean children with attention deficit hyperactivity disorder. Psychiatry Investig 2011;8:366-371.

2. Raman L, Pawashe AB, Ramalakshmi BA. Iron nutritional status of preschool children. Indian J Pediatr 1992;59:209-212.

3. Zimmermann MB, Hurrell RF. Nutritional iron deficiency. Lancet 2007; 370:511-520.

4. Morley CP. Disparities in ADHD assessment, diagnosis, and treatment. Int J Psychiatry Med 2010;40:383-389. 
5. Dopfner M, Steinhausen HC, Coghill D, Dalsgaard S, Poole L, Ralston SJ, et al. Cross-cultural reliability and validity of ADHD assessed by the ADHD Rating Scale in a pan-European study. Eur Child Adolesc Psychiatry 2006;15(Suppl 1):I46-I55.

6. Cepeda M. ADHD assessment: about the fifty percent referred to a child specialist. South Med J 2006;99:793-794.
7. Penberthy JK, Cox D, Breton M, Robeva R, Kalbfleisch ML, Loboschefski T, et al. Calibration of ADHD assessments across studies: a metaanalysis tool. Appl Psychophysiol Biofeedback 2005;30:31-51.

8. Dickerson Mayes S, Calhoun SL, Crowell EW. Clinical validity and interpretation of the Gordon Diagnostic System in ADHD assessments. Child Neuropsychol 2001;7:32-41. 